\title{
Applications of image processing in viticulture: A review
}

\author{
$\underline{\text { J. Whalley }}^{\mathrm{a}}$ and S. Shanmuganathan ${ }^{\mathrm{a}}$ \\ ${ }^{a}$ Geoinformatics Research Centre \\ AUT University, Auckland, New Zealand \\ Email: jwhalley@aut.ac.nz
}

\begin{abstract}
The economic importance of the wine industry worldwide is driving the development and application of innovative methods and technologies for monitoring vineyards. The harvest can significantly vary from year to year and also within the vineyard due to soil conditions, disease, pests, climate and variation in vineyard management practices. Current practices for yield and quality estimation are destructive, expensive, inaccurate and largely subjective. These factors make the production of high quality grapes for wine making challenging. Image analysis has the potential to provide an inexpensive, nondestructive way of capturing precise information about the entire vineyard.
\end{abstract}

This paper presents a review of recent research relevant to the application of digital image analysis to the management of vineyards focusing on the key challenges for in-field, on the vine, ground level image capture and analysis. The applications explored are:

Yield Estimation: Historically efforts have primarily been focused on the objective estimation of harvest yield. In order to estimate yield using image processing the grapes must be detected, segmented from the background and the number of clusters counted and the size of the cluster determined and this is typically how most estimation methods, using images, are approached. However for accurate yield forecasting berries within the cluster must be counted and the size of each berry determined. This is difficult to achieve due to the occlusion of berries. Many of the most successful grape detection methods have been developed, independently of the work on yield forecasting, for the automation of vineyard management activities such as harvesting and smart spraying rather than for yield estimation.

Quality Evaluation: Berry quality can be linked to visual properties such as the size, weight and colour of the berry. These properties have been proven to be indicators for ripeness and level of phenolics, flavonoids and sugar. To date most of the work in this area has focused on the processing of images of individual grapes in the laboratory. In the natural environment approaches must be able to cope with difficult and variable lighting conditions and with factors such as occlusion of the grapes within the cluster and by the canopy.

Disease Detection: While some work has been undertaken towards the detection of powdery and downy mildew and botrytis in grapes there is still significant scope for improvement. Detection in the field is difficult as the grapes may be covered by bloom and disease can exhibit different signs and symptoms depending on the grape variety and the stage of development of the disease. Moreover, more than one disease might be present. Current work does not address the issues of more than one disease or identification of the disease at various stages in its development. The most successful work to date has not been on in-field images.

Grape Phenology: The phenology of the grape vine is complex. Understanding the phenology of a given plant system is important in determining the ability of a region to produce a crop and knowledge of a plant's growth stages is advantageous as management practices (such as irrigation, pest and disease control and pruning) can be applied at optimum times in the vines growth cycle. Additionally, information regarding growth stages can be useful in forecasting crop yields and even quality.

The information gathered from automated, fast, accurate image analysis from in-field images could be used to design, train and validate simulations and forecasting models of vineyards and grape phenology. This type of research is at the forefront of climate research in which novel methods are required to monitor spatiotemporal physiological responses of food sources to changes in the environment.

The use of image processing to enhance vineyard practices and forecast the success and or quality of wine grapes is in its infancy. While much of the work to date is promising we have not yet achieved the "vineyard of the future", where image analysis (image and video) is a powerful tool that is adopted by viticulturists to inform the management of their vineyards.

Keywords: Image processing, classification, viticulture 


\section{INTRODUCTION}

Image processing of in-field and on the on-the-vine digital images has to overcome some unique challenges. Visually detecting grape berries is difficult. Moreover, the appearance of the grapes varies under different lighting conditions and in the case of white grapes, and of all grape varieties prior to véraison (the start of colour development), there is a lack of colour contrast between the canopy and the grapes. Additionally because grape berries occur in clusters many of the berries are occluded by other berries and by the leaves. Vineyard management practices such as vine training and pruning can also affect the quality of the images captured.

Since 1999, the use of remote image sensing techniques for precision viticulture (PV) has been the focus of much of the research in this area and while this work falls outside of the scope of this review it is worth while noting its applications in viticulture (see Hall et al., 2002). Typical applications of remote sensing involve producing a synoptic view of grapevine shape, size and vigour over the entire vineyard. However the use of remote sensing to capture the data is expensive and therefore unlikely to be adopted by vineyard managers until costs are reduced.

There have been a number of useful reviews of investigations into the use of image processing in other crops for example: food quality evaluation (Zheng et al., 2006), advances in disease detection (Patil and Kumar, 2011), automated spraying systems (Hong et al., 2012) and image processing in agriculture (Vibhute and Bodhe, 2012). While much of this work has some relevance to viticulture the challenges that exist in terms of colour contrast and occlusion of grapes by grape clusters and of clusters by the canopy do not exist in these other crops to the same extent (for example apple, cucumber and mango).

A recent review by Arnó et al. (2009) highlights the key challenges in the field of PV and notes that "In reality, research into $P V$ is still in its infancy, and to date relatively little has been published in this field". Techniques to improve vineyard management using in-field ground level digital imagery are also in their infancy. Most of the effort to date has been devoted to the location of grapes for robotic harvesting, pruning and smart spraying and to the estimation of harvest yield. However, some pioneering work has been undertaken to track and estimate the stage of development of the vine and fruit (phenology) and to visually detect disease. Disease detection is particularly difficult as disease can exhibit different signs and symptoms depending on the grape variety and the stage of development of the disease. Several diseases can be present at the same time and factors such as weather, nutrient deficiencies and pesticides can also produce signs that are similar to those of diseases.

What follows is a discussion of the work undertaken to date that is most relevant to the analysis of digital ground level and in-field imagery for viticulture.

\section{HARVEST YIELD AND QUALITY ESTIMATION}

Until recently methods for the estimation of yields in vineyards were dependent on the destructive harvesting of entire vines that had been randomly selected within the vineyard. For each selected vine the handharvested clusters/bunches were weighed and counted. From this information the average number of clusters per vine and the average weight per cluster could be calculated and this information could be extrapolated to the vineyard as a whole on the basis of the number of vines per acre. A sub sample of the harvested clusters was broken up, the number of berries counted and the berry size was measured using callipers.

Manually counting and weighing clusters is labour intensive, expensive, and inaccurate. Moreover the weight of clusters continues to increase up until the time of harvest. For these reasons vineyard managers have been unwilling to devote resources to crop yield estimation as the time for harvest approaches. A decade ago it was reported that there was still a lack of adoption of methods for yield information by vineyard managers despite the availability of better methods for crop forecasting (Dunn and Martin, 2003). In the past decade significant advances have been made in image capture hardware and in image processing techniques and these have provided a means of automating methods for determining the yield of crops.

In order to estimate yield in-field on the vine it is necessary to be able to detect the grape clusters. Significant advances in the reliable detection of clusters from digital images have been made with a view to its application in automated harvesting and spraying. Chamelat et al. (2006) developed a new method for detecting red grape clusters in outdoor images that used Zernike moments to describe grape shapes in addition to colour information. The images were subdivided into process blocks and a semi-supervised Support Vector Machine (SVM) was used for the recognition step. The method enabled the detection of grape clusters in any orientation and of any size with a $99 \%$ success rate. Later work by Reis et al. (2011) developed a method to detect red and white grapes in night captured images on the vine. Pixels are identified 
that fall within a set colour range then colour mapping is performed to generate a binary image, morphological dilation is then performed to fill in the gaps between pixels and regions that are small are discarded as false detections. The orientation of each region or bunch is determined by using pixel distribution and density around its centre in order to determine a probable location for the stem of the bunch. This method was reported to have a $9 \%$ false detection rate but was able to detect red and white grapes in all images. A recent advancement in the segmentation and identification of individual grapes from the image of a cluster used a circular Hough transform to detect berries, including those partially occluded, and segmented them from the background (Murillo-Bracamontes et al., 2012). False detections were then excluded using colour information. To our knowledge these approaches have not been extended in order to estimate yield or quality of harvest.

In 2004 digital photographs of the canopy of Cabernet Sauvignon grapevines were captured as the vine was progressively harvested (Dunn and Martin, 2004). The images were captured in-field on the vine using a white screen behind the canopy. Colour features were extracted from the RGB (red, green, and blue) colour space and a manually set threshold and tolerance values were set in order to select the "fruit" pixels for a single image. These settings were retained and used in the analysis of the remaining images. The segmented fruit pixels were then counted. They found that using RGB colour features they could successfully segment the berry clusters from other parts of the canopy and concluded that yield estimation of grapevines using digital image analysis was feasible. However, although this preliminary and pioneering work looks promising the canopy structure chosen was simple, with well exposed fruit and vertical shoot positioning. It is unlikely that using solely RGB features would be adequate for white grape varieties where the colour of the berry is similar to that of the canopy especially in vineyards with alternative trellising systems or vineyards that use minimal pruning or hedging training systems.

The most accurate method of crop estimation in machine or minimal pruned vineyards is based on average berry weights during the season as opposed to the traditional approach of cluster weights typically used in hand-pruned vineyards (Poole et al., 1993). One approach reported for counting individual Pinot Noir berries used a common flatbed scanner to take images of samples of loose grapes and used simple image processing techniques in order to count the grapes (Battany, 2008). The captured greyscale images were converted to binary images then joined berries were separated using watershed segmentation (Vincent and Soille, 1991) and finally the berries were counted using a size threshold to eliminate debris. While this approach leads to a relatively accurate berry count, utilizes low cost equipment and is faster and more accurate than manually counting berries, it still requires destructive harvesting and in practice would require sampling which may result in inaccuracies in yield estimation.

Nuske et al. (2011) developed an automated computer vision method to detect and count grape berries. Their method provided a precise, non-invasive and rapid means of estimating yield to within $9.8 \%$ of the actual crop yield. To our knowledge, this technique is the first reported method that was able to reliably estimate both white and red grape yields because it relies solely on colour contrast. A conventional visible light camera was mounted on a small vineyard vehicle which enabled a vineyard in its entirety to be surveyed. The reported algorithm has three separate stages. The first stage detects the berry locations using a radial symmetry transform. Points with a high level of radial symmetry are identified as grape berry centres. The second stage classifies the detected points which are most likely to be grapes using a combination of colour and texture features and a k-Nearest Neighbour classifier. The third stage attempts to remove the small set of remaining false positive detections by grouping berries into clusters. Because grape berries naturally occur in clusters if a detected berry does not have at least 5 berries in close proximity then it is eliminated.

An extension of this work that utilised calibration data obtained either from previous harvests or a small set of destructive handpicked samples at the time of imaging was recently reported (Nuske et al., 2012). Using this approach they were able to achieve a $4 \%$ improvement in the accuracy of the yield estimate using previous harvest calibration and $3 \%$ with the handpicked samples.

In viticulture berry number per cluster, berry size and weight are key parameters for yield estimation and are also employed as indicators for grape and wine quality. Research has provided evidence for the relationship between berry size and quality (Roby et al., 2004) where small berries correlate with a lack of water and berry size heterogeneity indicates a lack of-homogenous maturation. Rabatel and Guzard (2007) introduced an image processing approach to estimating berry size of vine grapes, in the cluster, by image acquisition in the field as a way of estimating the growth stage and potential quality of the vine grapes. An image of a cluster of berries with almost identical colour features relies on contours, detected as luminance discontinuities, for berry separation. Typically in the work on yield estimation (see the previous section) edge detection is reliant on a threshold but in this work a watershed algorithm was adopted that was less dependent on a threshold. An ellipse model was then fitted to a set of compatible contour arcs using Fitzgibbon's least 
square algorithm (1999). Although the ellipse model is well suited to berry profiles a third of the visible berries on the vine were not detected. The authors concluded that by introducing adaptive thresholding in the watershed step and by model fitting using a multivariate semi-supervised classification system the size estimation would be accurate enough to evaluate the grape quality. To our knowledge the proposed extension of this work has not been reported. More recent work by Tardaguila et al. (2012) reliably determined the size and weight of grape berries by extracting the morphology of a grape using Freeman's chain code algorithm (1961). However, this method was destructive and developed for individual grapes, not clusters, under laboratory conditions. At this point in time although significant progress has been made there is still not a practical method for the accurate and rapid measurement of grape size and weight in the field (on the vine) that would allow grape growers to assess at various point during the growing season the ripeness and quality of the crop throughout the vineyard.

\section{DISEASE IDENTIFICATION}

In a 2005 survey of grape growers in Washington State (USA) Powdery Mildew and Grape Leaf Roll virus were identified as the most problematic diseases for grape growers. The survey also identified Eutypa dieback, Botrytis bunch rot (botrytis) and Crown gall as problematic. Fungal diseases in particular cause severe problems for wine grapes which are particularly susceptible to Powdery Mildew, Downy Mildew and Botrytis. Research has shown that infection levels of Downy Mildew as low as 1-5\% can taint the flavour of wine (Strummer et al., 2005). Botrytis can decrease grape yield and wine quality (Nair and Hill, 1992) to such an extent that Australasian wineries often impose price penalties if more than $3-5 \%$ of the harvested crop is affected by botrytis (Hill et al., 2010).

In the past downy mildew has been controlled with extensive use of fungicides. This is not only a costly practice but also affects the public perception of the crop and in the case of wine has a negative influence on the quality of the vintage. These negative factors have led to breeding programs which integrate wild relatives to traditional wine cultivars in order to introduce genetic resistance in an attempt to eliminate or at least reduce the need for fungicides.

Assessment of resistance to grapevine downy mildew is typically performed using subjective visual inspection. In an early study mature leaves, with oily spots, exhibiting symptoms of downy mildew were digitally photographed on the vine and examined using anaySIS 3.0 (Soft Imaging System GmbH, 1998) to count the number of spots and determine the severity and intensity of the infection of eight different clones of the grape cultivar Albariño (Boso et al., 2004). The work by Boso et al. confirmed that image processing can provide a means of rapid, reliable and quantitative early detection of these diseases that if developed further could, in the future, reduce preventative spraying practices. Since this early work significant progress has been made in the used of image processing approaches to detect various diseases in other crops (for example: Corkidi et al., 2006; Bock et al., 2008). While this work was successful, it focused on the development of techniques that quantified the fungal growth and focused on monitoring fungal structures rather than symptoms and did not provide information about the development of the pathogen.

Peresotti et al. (2011) developed a semi-automated image processing method to measure the development of the downy mildew pathogen by quantifying the sporulation of the fungi on the grapevine with a view to evaluating the resistance of different grape vine cultivars. They artificially inoculated small discs of a grapevine leaf and then took photographs at a constant focal length in the petri dish over several days. Using ImageJ software (http://rsbweb.nih.gov/ij/), the colour of the images was balanced in order to provide a uniform white/green ratio across the images. Then rolling ball background subtraction (see: Sternberg, 1983) was used to isolate the leaf disc. In order to quantify the sporulation the image was converted to 8-bit format using median-cut colour quantization (Heckbert, 1982) and then the contrast was adjusted to enhance the white sporulated area in the leaf. The area to be measured was then selected using ImageJ auto-thresholding (a variation of the IsoData algorithm (Ridler and Calvard, 1978)), and a manually set threshold. The sporulated pixels were then counted. This method was found to be accurate, fast and reproducible and the development of the pathogen could be observed. However, this approach requires leaf disc bio-assay of selected vines within the vineyard. Ideally this sort of quantification and monitoring of development of disease would be done in the vineyard under natural conditions in a non-invasive manner.

In 2008, Meunkaewjinda et al. classified grape leaves into one of three classes; scab disease, rust disease or no disease using an image processing approach. Their system consisted of three key stages namely, grape leaf segmentation, diseased leaf segmentation and disease analysis and classification. They used images of grape clusters on the vine and leaves were detected using a hybrid system consisting of a self-organising feature map (SFOM) and back propagation neural network to recognise leaf colour. The resulting information was used to segment the grape leaf pixels from the image. Diseased leaf segmentation was performed with a 
modified SFOM for which the parameters were optimised using a genetic algorithm. The segmented image was then subjected to a Gabor wavelet filter that allowed the system to analyse the disease colour features more efficiently and the SVM then used to classify the grape leaf disease. There were some limitations noted in the segmentation stages due to the extraction of ambiguous colour pixels from the background of the image. However, despite these limitations the system gave good results and demonstrated the potential for automatic diagnosis of grapevine diseases

More recently Li et al. (2012) reported an image recognition method for the diagnosis of vines with downy mildew and powdery mildew disease. The aim was to provide a reliable method of disease identification for grape growers. Images were pre-processed using nearest neighbour interpolation to compress the image prior to removal by a median filter and finally diseased regions were segmented via a K_means clustering algorithm. Then a SVM classifier was evaluated for performance in disease recognition. Thirty one features including colour, texture and morphologically based features and a linear kernel SVM gave the best recognition results with recognition rates of $90 \%$ for downy mildew and $93 \%$ for powdery mildew.

Given the significant impact of disease on wine quality it is surprising that little work has been undertaken to automate the early detection of these diseases in vineyards. In order for in-field detection methods to be adopted they must be unsupervised, cost effective, and reliable and produce results in real-time. Obviously in-field detection has many challenges that we have previously identified in terms of image capture. But if we can develop an accurate and reliable disease identification technique it would allow for the automation of spraying and targeting of affected areas and reduce the cost of disease prevention. Additionally it could help growers to empirically identify stock for further breeding in order to cultivate disease resistant stock.

\section{PHENOLOGY}

The phenology of the grapevine is a complex process that is dependent on many environmental factors including climate and vineyard management practices. Identification of the growth stages of the vine is essential to measure the effects of local environmental and climatic change on the development of the grapes and subsequently the quality of the wine. From season to season varying weather conditions can significantly affect the development of the vines and fruit and therefore impact on vineyard management planning and practices.

Identifying the stages of development and their occurrence using traditional methods is time consuming and to some extent subjective. The most widely adopted system for the identification of the phenology of the grapevine was developed by Lorenz et al. in 1995 (for an English translation see: Coombes, 1995). Plant development characterisation using image processing methods is a relatively new and growing field. Some preliminary work has been reported that uses images to monitor and estimate the development stage(s) of grape vines.

Rodríguez-Pulido et al. (2012) reported a system to estimate the ripeness of red grapes. They monitored the visual changes in berries by capturing images of the grapes from the onset of véraison (the ripening period). The development of the grapes can be determined visually by increase in size and colour: unripe berries are green, as they develop the colour turns lighter and pink areas appear and then they turn to purple until the purple appears almost black. These visual changes in colour and morphology are closely linked to chemical changes that occur within the fruit during ripening. For red wine in particular these visual changes are closely linked to the level of phenolics which give the wine intensity and stability.

The analysis employed known algorithms. The first stage was segmentation in the HSI colour space using a histogram based segmentation method (Cheng et al., 2001) with morphological restrictions based on the detected object's Feret diameter. Rodríguez-Pulido et al. (2012) then applied a threshold based on the brightness of the colour in order to estimate the veraison level of the grapes. This threshold was determined experimentally based on the RGB values of the grape images pre- and post-véraison. The véraison level for each cluster was calculated as the percentage of berries that met the threshold $((\mathrm{R}+\mathrm{G}+\mathrm{B}) / 3, \geq 90 \rightarrow$ prevéraison, $<90 \rightarrow$ post- véraison). This method is reported to be an effective and objective means of estimating the véraison level of the grapes as well as the size of the grapes. Both of these factors are routinely assessed by grape growers during the development of vines each season in order to determine time of harvest.

\section{CONCLUSION}

Recent advances in digital image processing and computer vision techniques have the potential to enhance vineyard management and bring the more scientifically managed vineyard of the future one step closer to becoming a reality. There are still many challenges in terms of developing fast, accurate and generic 
algorithms that will assist in the detection of disease and location of grapes in real time without the need for supervision (e.g. User defined thresholds).

We have yet to develop a system that can track and predict the phenological stages of the development of the vine and its fruit from season to season. Given the vulnerability of vineyards to environmental factors that ability to track environmental factors (weather, disease, pests and weeds) and vineyard management practices and to measure and forecast their impact on the growth and development of the vine and fruit in an empirical way would be invaluable. The information gathered could be used to design, train and validate simulations and forecasting models of vineyards and grape phenology.

Image processing potentially has a large role to play in the automation of vineyard management. Applications include: the robotic pruning and spraying of the vines and harvesting of the fruit, the scheduling of treatments (fertilisation and pest/disease control measures), harvest and irrigation, the evaluation of new cultivars and the effect of vineyard management practices.

\section{REFERENCES}

Arnó, J., Marínez-Casasnovas, J.A., Ribes-Dasi, M., and Rosell, J.R. (2009). Review. Precision Viticulture. Research Topics, challenges, and opportunities in site-specific vineyard management. Spanish Journal of Agricultural Research, 7(4), 779-790.

Battany, M. (2008). A Practical Method for Counting Berries based on Image Analysis. $2^{\text {nd }}$ Annual National Viticulture Research Conference, University of California, Davis, USA, 4-5, July 9-11, 2008.

Boso, S., Santiago, J.L., and Martínez, M.C. (2004). Resistance of Eight Different Clones of the Grape Cultivar Albariño to Plasmopara viticola. Plant Disease, 88(7), 741-744.

Chamelat, R., Rosso, E., Choksuriwong, A., Rosenberger, C., Laurent, H., and Bro, P. (2006). Grape Detection by Image Processing Symposium conducted at the meeting of IECON 2006.

Cheng, H.D., Jiang, X.H., Sun, Y., and Wang, J. (2001). Color image segmentation: advances and prospects. Pattern Recognition, 43, 2259-2281.

Dunn G.M., Martin S.R. (2004). Yield prediction from digital image analysis: A technique with potential for vineyard assessments prior to harvest. Aust. J. Grape Wine Res., 10, 196-198.

Dunn, G.M., and Martin S.R. (2003). The current status of crop forecasting in the Australian wine Industry. ASVO seminar series: Grape growing at the Edge, Tanunda, Barossa Valley, SA, 4-8, July 2003.

Fitzgibbon, A., Pilu, M., and Fischer, R.B. (1999). Direct Least Squares Fitting of Ellipses. IEEE Transactions on Pattern Analysis and Machine Intelligence, 21, 476-480.

Freeman, H. (1961). On the encoding of arbitrary geometric configurations. IRE Transactions on Electronic Computers. EC-10, 260-268.

Hall, A., Louis, J., and Lamb, D. (2003). Characterising and mapping vineyard canopy using high-spatialresolution aerial multispectral images. Computers \& Geosciences, 29(7), 813-822.

Hall, A., Lamb, D.W., Holzapfel, B., and Louis, J. (2002). Optical remote sensing applications in viticulture - a review. Aust. J. Grape Wine Res., 8, 36-47.

Heckbert, P. (1982). Color image quantization for frame buffer display, 9th annual conference on Computer graphics and interactive techniques SIG-GRAPH'82, ACM Press, New York, NY, USA, 297-307

Hill, G.N., Beresford R.M., and Evans, K.J. ( 2010). Tools for accurate assessment of botrytis bunch rot (Botrytis cinerea) on wine grapes. New Zealand Plant Protection, 63, 174-181.

Hong, S., Minzan, L. and Zhang, Q. (2012). Detection system of smart sprayers: Status, challenges, and perspectives. Int. J. Agric \& Biol Eng, 5(3), 1-15.

Li, G.L., Ma, Z.H., and Wang, H.G. (2012). Image Recognition of Grape Downy Mildew and Grape Powdery Mildew Based on Support Vector Machine. Computer and Computing Technologies in Agriculture, In V. D. Li and Y. Chen (Eds), Springer Berlin Heidelberg. 370, 151-162.

Meunkaewjinda, A., Kumsawat, P., Attakitmongcol, K., and Srikaew, A. (2008). Grape leaf disease detection from color imagery using hybrid intelligent system. Symposium conducted at 5th ECTI-CON 2008., 513516, 14-17 May, 2008. 
Whalley and Shanmuganathan, Applications of image processing in viticulture: A review

Murillo-Bracamontes E.A., Martínez-Rosas, M.E., Miranda-Velasco, M.M., Martínez-Reyes, H.L., Martínez -Sandoval, J.R., and Carvantes-de-Avila, H. (2012). Implementation of Hough transform for fruit image segmentation. Procedia Engineering, 35, 230-239.

Nair N.G., and Hill G.K. (1992). Bunch rot of grapes caused by Botrytis cinerea. Plant diseases of international importance, III: Diseases of fruit crops. Prentice-Hall, Inc., NJ, USA. 147-169.

Nuske, S., Achar, S., Bates, T., Narasimhan, S., and Singh, S. (2011). Yield Estimation in Vineyards by Visual Grape Detection. IEEE/RSJ International Conference on Intelligent Robots and Systems (IROS); San Francisco, CA, USA, 2352-2358, 25-30 September, 2011.

Nuske, S., Gupta, K., Narasimhan, S., and Singh, S. (2012). Modeling and Calibrating Visual Yield Estimates in Vineyards. $8^{\text {th }}$ International Conference on Field and Service Robotics, Miyagi, Japan, 16-19 July, 2012.

Patil, J.K, and Kumar, R. (2011). Advances in image processing for detection of plant diseases. Journal of Advanced Bioinformatics Applications and Research, 2(2), 135-141.

Peressotti, E., Duchêne, E., Merdinoglu, D., and Mestre, P. (2011). A semi-automatic non-destructive method to quantify downy mildew sporulation. Journal of Microbiological Methods, 84, 265-271.

Poole, R.M., Dunst, R.E., Crowe, D.C., Hubbard, H., Howard, G.E., and DeGolier, G. (1993). Predicting and controlling crop of machine and minimal pruned grapevines. $2^{\text {nd }}$ Shaulis Symposium - Pruning and mechanisation of crop control. Fredonia, NY, NY State Agric. Exper. Sta. Spec. Publ., 31-45.

Rabatel, G., \& Guizard, C. (2007). Grape berry calibration by computer vision using elliptical model fitting. 6th European Conference on Precision Agriculture.

Ridler, T.W., and Calvard, S. (1978), Picture thresholding using an iterative selection method. IEEE Transactions on Systems, Man and Cybernetics, 8, 630-632.

Ries, M.C., Morais, R., Pereira, C., Soares, S., Valente, A., Baptista, J., Ferreira, J.S.G., and Cruz, J.B. (2011). Automatic Detection of White Grapes Using Image Processing. Soft Computing in Industrial and Environmental Applications, 87, 19-27.

Roby, G., Harbertson, J.F., Adams, D.A., \& Matthews, M.A. (2004). Berry size and vine water deficits as factors in winegrape composition: Anthocyanins and tannins. . Aust. J. Grape Wine Res., 10, 100-107.

Rodriguez, F.J., Gomez-Robledo, L., Melgosa, M., Gordillo, B., Gonzalez-Miret, M.L., and Heredia, J. (2012). Ripeness Estimation of grape berries and seeds by image analysis. Computers and Electronics in Agriculture, 82, 128-133.

Sternberg, S. R. (1983). Biomedical Image Processing. Computer, 16(1), 22-34.

Stummer, B.E., I. L. Francis, I.L., Zanker, T., Lattey, K.A., and Scott, E.S. (2005). Effects of powdery mildew on the sensory properties and composition of Chardonnay juice and wine when grape sugar ripeness is standardised Aust. J. Grape Wine Res., 11(1), 66-76.

Tardaguila, J., Diago, M.P., Millán, B., Blasco, J., Cubero, S. García-Navarrete, O.L., and Aleixos, N. (2012). Automatic estimation of the size and weight of grapevine berries by image analysis. CIGR-Ageng. http://wcigr.ageng2012.org/images/fotosg/tabla_137_C1735.pdf

Vibhute, A., and Bodhe, S.K. (2012). Applications of Image Processing in Agriculture: A Survey. International Journal of Computer Applications, 52(2), 34-40.

Vincent, L., and Soille, P. (1991). Watersheds in digital spaces: an efficient algorithm based on immersion simulations. IEEE Trans. Patt. Anal. Mach. Intell., 13(6), 583-598.

Zheng, C., Sun, D-W., and Zheng L. (2006). Recent developments and applications of image features for food quality evaluation and inspection - a review. Trends in Food Science \& Technology, 17, 642-655. 